\title{
Análise espacial da mosca-negra em sistema agroflorestal de citros
}

\author{
Anderson Gonçalves da Silva'*, Paulo Roberto Silva Farias' , Arlindo Leal Boiça Junior², \\ Bruno Gonçaves Lima², Nara Helena Tavares da Ponte², \\ Raphael Coelho Pinho², Ronny Sobreira Barbosa ${ }^{3}$ \\ 'Universidade Federal Rural da Amazônia, Belém, PA, Brasil \\ 2Universidade Estadual Paulista Júlio de Mesquita Filho, São Paulo, SP, Brasil \\ 3Universidade Federal do Piaú, Campus Professora Cinobelina Elvas, Bom Jesus, PI, Brasil \\ *Autor correspondente; e-mail: agroanderson.silva@yahoo.com.br
}

\begin{abstract}
Resumo
O objetivo deste trabalho foi caracterizar a distribuição espacial da mosca-negra-dos-citros (Aleurocanthus woglumi) em pomar de citros em plantio agroflorestal no Estado do Pará. A área experimental está localizada no município de Capitão Poço, região nordeste do Estado do Pará, onde foram realizadas amostragens mensais, avaliando a presença ou ausência da praga na área experimental. De cada ponto de amostragem (planta) obteve-se o valor da variável e as coordenadas (latitude e longitude). Através dos parâmetros dos modelos do semivariograma, foram interpolados os levantamentos através da krigagem que nos forneceu mapas da distribuição espacial mostrando as áreas de maior e menor infestação de mosca negra. Os resultados mostram que a distribuição espacial da praga dá-se, predominantemente, em agrupamentos com dependência espacial descrita pelo modelo esférico, formando reboleiras de 15,5 a $34 \mathrm{~m}$ (alcance do modelo).
\end{abstract}

Palavras-chaves: Aleurocanthus woglumi Ashby 1915, Aleyrodidae, Citrus sinensis, Tectona grandis, geoestatística

\section{Spatial analysis of blackfly in agroforestry system of citrus}

\begin{abstract}
The aim of this study was to characterize the spatial distribution of citrus black fly (Aleurocanthus woglumi) in citrus orchard in agroforestry plantation in Pará State, Brazil. The experimental area is located in Capitão Poço, Northeastern Pará. Twelve samples were taken monthly where the presence or absence of the pest in the experimental area were evaluated. From each sampling point (plant) we obtained the value of the variable and the coordinates (latitude and longitude). By the parameters of semivariogram models the surveys were interpolated by kriging method which provided us spatial distribution maps of the areas of higher and lower infestation of the black fly. The results showed that the spatial distribution of black fly takes place predominantly in clusters with spatial dependence described by the spherical model, forming clusters from 15.5 to $34 \mathrm{~m}$ (range of the model).
\end{abstract}

Keywords: Aleurocanthus woglumi Ashby 1915, Aleyrodidae, Citrus sinensis, Tectona grandis, geostatistics 


\section{Introdução}

Os sistemas agroflorestais (SAFs) são definidos como técnicas alternativas de uso da terra, que implicam na combinação de espécies florestais com culturas agrícolas (Yared et al., 1998). Dentre as espécies florestais que são utilizadas consorciadas com citros, a Teca (Tectona grandis Linn. F.) merece destaque por ser espécie arbórea de alto valor comercial (Moeira et al., 2006).

Dentre os principais problemas fitossanitários do estado, a mosca-negra-doscitros, Aleurocanthus woglumi Ashby, 1915 (Hemiptera: Aleyrodidae) destaca-se por acarretar danos diretos e indiretos ás plantas cítricas, além de se constitui praga quarentenária presente ou A2 de alerta máximo, restringindo o comércio com outras regiões livres da mesma (MAPA, 2010). Nativa da Ásia, a mosca-negrados-citros apresenta-se amplamente distribuída geograficamente só não tendo relatos de sua ocorrência em regiões do continente Europeu e nos polos (Silva, 2005; Silva et al., 201 1a). No Brasil, sua primeira ocorrência foi relatada no Estado do Pará em 2001 (Silva, 2005), e hoje já se encontra disseminada pelos estados por vários estados brasileiros (Pena et al., 2008; Correia et al., 2011; Monteiro et al., 2012). Na região amazônica a praga encontra condições ideais de desenvolvimento, apresentando um rápido ciclo evolutivo (Silva et al.,, 2011 a).

Para estudar a dependência espacial, a geoestatística é a ferramenta mais adequada, pois considera a posição no espaço da variável em estudo, permitindo quantificar essa dependência espacial entre amostras coletadas em campo e utilizar a mesma para construção de mapas (Leibhold et al., 1993). Com isso, é possível determinar a dependência espacial da praga, através da elaboração de semivariogramas que são ajustados a um modelo que fornece o raio de agregação.

A partir desse semivariograma, é feita a elaboração de mapas que mostram como ocorre a expansão da praga na área (Leal et al., 2010). Dessa forma, a incorporação de procedimentos geoestatísticos baseados em técnicas de modelagem espacial por semivariogramas e estimação via krigagem são ferramentas importantes, podendo ser utilizadas para determinar, dentre outros parâmetros, a distribuição espacial, elaboração de métodos seguros de amostragem e, principalmente, controle localizado deste aleirodídeo no campo.

Alguns estudos a fim de verificar - comportamento espacial de pragas de importância econômica já foram feitos como os de Farias et al. (2002), Farias et al. (2004), DinardoMiranda et al. (2007), Maia (2008) e Leal et al. (2010), todos comprovando a eficiência do uso da ferramenta geoestatística para melhoria no manejo dos organismos envolvidos. No entanto, para o estudo do A. wuglumi, os trabalhos são incipientes nas pouca coisa foi feita em condições amazônicas.

Dada a importância desta praga e a falta de estudos básicos para se implementar o manejo adequado da mesma em sistemas agroflorestais em condições de campo, objetivou-se com $\circ$ presente trabalho caracterizar a distribuição espacial da moscanegra-dos-citros (A. woglumi) em pomar de citros consorciado com teca em sistema agroflorestal, no Estado do Pará utilizando a metodologia geoestatística.

\section{Material e Métodos}

O estudo foi desenvolvido no município de Capitão Poço, responsável por aproximadamente $71 \%$ da produção citrícola paraense (IBGE, 2012), distante 200 km de Belém, PA.

A área experimental é constituída por um pomar de laranjeiras da variedade "Pêra Rio" (Citrus sinens [L.] Osbeck), enxertadas em limão cravo (Citrus limonia Osbeck), plantadas em espaçamento de $5 \times 7 \mathrm{~m}$, apresentando sete anos de idade; distribuídas em 32 fileiras, com 52 plantas de citros por linha de plantio. Possuindo 1.664 plantas, totalizando $58.240 \mathrm{~m}^{2}$, o que corresponde a $89,9 \%$ da área total do sistema agroflorestal, com densidade de plantio de 285 laranjeiras por hectare. Na área, a distribuição das plantas de Teca se da a cada três linhas de plantio de citros totalizando 11 linhas com plantas de Teca. Essas estão distribuídas na linha de plantio a cada três plantas de citros, totalizando 187 plantas de Teca o que corresponde a 10,1\% 
da área total do pomar.

- levantamento populacional foi realizado em condições de campo em 100\% das plantas do talhão (sistema de avaliação em varredura), avaliando-se a presença ou ausência de ninfas e adultos vivos de A. woglumi; avaliando os quadrantes norte, sul, leste e oeste das copas observando principalmente brotações novas (onde geralmente são encontrados os adultos) e também a parte abaxial das folhas, através de vistorias minuciosas da parte inferior para a superior de cada planta, onde se encontram todas as fases imaturas da praga, anotando-se presença com o número um (1) ou ausência com o número zero (0). A avaliação de ninfas vivas foi realizada no campo, com auxilio de lupas. Os índices de infestação de A. woglumi para a presente área experimental, bem como, os valores das variáveis climáticas como precipitação pluviométrica $(\mathrm{mm})$ e temperatura $\left({ }^{\circ} \mathrm{C}\right)$ encontram-se em trabalho de Silva et al. (2011a). Realizaram-se 12 amostragens durante o período de 2008 a 2009. Na área experimental não se utilizou inseticida no período das avaliações de A. woglumi

\section{Análise Geoestatística dos Dados}

Os procedimentos a serem descritos seguem a metodologia de Vieira et al. (1983) e utilizam-se da informação da posição da amostra e o valor que a variável (infestação de mosca-negra-dos-citros) assumiu em cada ponto (planta). Desta forma, de cada ponto de amostragem obteve-se o valor da variável e as coordenadas (longitude e altitude) do ponto onde foi realizada a amostragem.

\section{Semivariograma Experimental}

obtenção do semivariograma é o passo mais importante no procedimento de uma análise geoestatística, aja visto, que o modelo escolhido será utilizado no processo da krigagem (Mapas de Contornos) e influenciará todos os resultados interpolados e as conclusões.

O semivariograma é estimado por:

$$
\begin{aligned}
\gamma^{*}(h) & =\frac{1}{2 N(h)} \sum_{i=1}^{N(h)}\left[Z\left(x_{i}\right)-Z\left(x_{i}+h\right)\right]^{2} \\
& \text { onde } N(h) \text { é o número de pares }
\end{aligned}
$$

experimentais de valores medidos $Z(x i), Z(x i+h)$, separados por um vetor $h$. O gráfico de $?^{*}(h)$ "versus" os valores correspondentes de $h$, chamado semivariograma, é uma função do vetor $h$ e, portanto, depende da magnitude e direção de $h$.

Para melhor definição dos parâmetros variográficos, foram feitas várias tentativas com ajuste aos modelos esférico e exponencial, utilizando distância (alcance) padrão de $60 \mathrm{~m}$ para modelar os semivariogramas da área experimental.

$$
\begin{aligned}
& \text { Modelo Esférico } \\
& \gamma(h)=C_{0}+C_{1}\left[\frac{3}{2}\left(\frac{h}{a}\right)-\frac{1}{2}\left(\frac{h}{a}\right)^{3}\right], \quad 0<h<a \\
& \gamma(h)=C_{0}+C_{1}, \quad h \geq a
\end{aligned}
$$

O modelo esférico é um dos modelos mais utilizados. É obtido selecionando-se os valores do efeito pepita, $C_{0^{\prime}}$ e do patamar, $C_{1^{\prime}}$ depois passando-se uma reta que intercepte o eixo y em $C_{1}$ e seja tangente aos primeiros pontos próximos de $\mathrm{h}=0$. Essa tangente cruzará o patamar à distância, $a^{\prime}=2 / 3$ a. Assim, o alcance, $a$, será $a=3 a^{\prime} / 2$. O modelo esférico é linear até aproximadamente 1/3 a.

\section{Modelo Exponencial}

Outro modelo bastante utilizado:

$$
\gamma(h)=C_{0}+C_{l}\left[1-\exp \left(-3 \frac{h}{a}\right)\right], \quad 0<h<d
$$

onde d é a máxima distância na qual o semivariograma é definido. Uma diferença fundamental entre o modelo exponencial e o esférico é que o exponencial atinge o patamar apenas assintóticamente, enquanto que o modelo esférico o atinge no valor do alcance. $\bigcirc$ parâmetro a é determinado visualmente como a distância após a qual o semivariograma se estabiliza. Os parâmetros $C_{0}$ e $C_{1}$ para o modelo exponencial são determinados da mesma maneira que para o esférico (Farias et al., 2002).

$$
\text { Modelo Aleatório (Efeito Pepita Puro) }
$$

$$
\gamma(h)=C, \quad \text { para qualquer } \mathrm{h}
$$

À medida que aumenta a 
descontinuidade na origem do semivariograma, mais aleatório é o fenômeno que originou a variável em análise. Esta característica decorre de uma provável regionalização, inferior à escala de trabalho da malha de amostragem e/ou às variações espúrias associadas com a coleta e medição das amostras (Farias et al., 2002).

\section{Krigagem}

Muitas vezes o interesse da análise não se esgota em modelar a estrutura de variabilidade. Em diversas situações o interesse está na estimação de valores em pontos não amostrados, seja por um interesse local ou pela intenção de obter um detalhamento da área que vai além do permitido pela amostra. Nestes casos é preciso lançar mão de algum interpolador (preditor) dentre os existentes na literatura (Farias et al., 2002). Dentre esses, a krigagem é considerado um estimador ótimo por não ser tendencioso e apresentar variância mínima.

Supondo-se que se queira estimar valores $z^{*}$, para qualquer local, $x_{0}$, onde não se tem valores medidos, e que a estimativa deve ser uma combinação linear dos valores medidos, tem-se:

$$
z *\left(x_{0}\right)=\sum_{i=1}^{N} \lambda_{i} z\left(x_{i}\right)
$$

onde $N$ é o número de vizinhos medidos, $Z\left(x_{i}\right)$, utilizados na estimativa da propriedade e $\lambda_{i}$ são os ponderadores aplicados a cada $Z\left(x_{i}\right)$, os quais são selecionados de forma que a estimativa obtida seja não tendenciosa. Os valores estimados por krigagem foram utilizados no programa SURFER 8.0 para confecção dos mapas.

\section{Resultados e Discussão}

Observou-se para o presente estudo que, com exceção do mês de fevereiro que apresentou distribuição com dependência espacial descrita por um modelo aleatório, todos os meses avaliados apresentaram dependência espacial descrita pelo modelo esférico, demonstrando que a mosca-negra-dos-citros apresenta-se de forma agregada no campo, formando reboleiras na área experimental (Tabela 1).
Os meses que apresentaram menor alcance foram os de novembro e julho com 15,5 $\mathrm{m}$ de alcance, o que corresponde a uma área de agregação de $754 \mathrm{~m}^{2}$, compreendendo em torno de 21 plantas. Os maiores alcances foram observados nos meses de abril e agosto com respectivos alcances de 28 e $34 \mathrm{~m}$, formando áreas de agregação de 2.462 e $3.630 \mathrm{~m}^{2}$, compreendendo 70 e 104 plantas respectivamente, para os referidos meses (Tabela 1).

A distribuição agregada foi a que melhor representou o comportamento da moscanegra-dos-citros na área experimental. Maia (2008) em estudos na mesma região do presente experimento observou distribuição agregada da mosca negra em todas as avaliações. Este tipo de distribuição também foi encontrado em estudos de Soto et al. (2002), para a distribuição dos aleirodídeos Aleurothrixus floccosus (Maskell), Dialeurodes citri (Ashmead) e Parabemisia myricae (Kuwana) em citros na Espanha, com A. floccosus apresentando maior agregação que as outras duas espécies. Argov et al. (1999) estudando a dispersão de D. citri em pomares cítricos em Israel, observaram que a distribuição da praga se deu de forma agregada em todas as suas fases de desenvolvimento.

Estudos de Silva et al. (201 1 b), na mesma região, porém em plantio convencional de citros, encontraram distribuição agregada da moscanegra na área de estudo, formando "reboleiras" de 8,5 a $30 \mathrm{~m}$ (alcance do modelo). Nota-se, que para o presente estudo, foram obtidos alcances maiores, o que pode ser explicado pela presença de plantas de Teca na área.

Este modelo de distribuição apresentado pode está associado a uma autonomia de vôo reduzido propiciado principalmente pela presença de plantas de Teca na área, a moscanegra-dos-citros apresenta um voo curto (Dowel \& Fitzpatrick, 1978), facilitado principalmente pelo vento que atua auxiliando sua dispersão no pomar. Com a redução deste fator na área a praga disseminou-se de planta para planta principalmente na linha de plantio, como fica bem evidenciado nos mapas de krigagem (Figura 2). Essa disseminação da praga, principalmente na linha de plantio, também foi 
observada em trabalho de Silva et al. (2011b), avaliando o A. woglumi em área convencional de citros. O exposto também foi observado por Pasek (1988), que menciona que ambos os movimentos horizontais e verticais dos insetos são afetados pela introdução de espécies florestais em sistemas agroflorestais. Root (1973) acrescenta que essas plantas introduzidas atuam fisicamente como barreira mecânica para a dispersão do inseto-praga, fato já verificado para pulgões, tripes, moscas e pequenos besouros (Levin, 1973).

Tabela 1. Parâmetros dos semivariogramas das amostragens de mosca-negra-dos-citros (A. woglumi), ajustados aos modelos.

\begin{tabular}{|c|c|c|c|c|c|c|c|}
\hline \multirow{2}{*}{$\begin{array}{c}\text { Data de } \\
\text { amostragem }\end{array}$} & \multicolumn{3}{|c|}{ Parâmetros* } & \multirow{2}{*}{ Modelo } & \multirow{2}{*}{$\begin{array}{l}\text { Área }\left(\mathrm{m}^{2}\right)^{a} \\
\text { da reboleira }\end{array}$} & \multirow{2}{*}{$\begin{array}{l}\text { Número de } \\
\text { Plantas }\left(\mathrm{m}^{2}\right)^{\mathrm{b}} \\
\text { na reboleira }\end{array}$} & \multirow{2}{*}{$K^{c}$} \\
\hline & $C_{0}$ & $C_{1}$ & $a(m)$ & & & & \\
\hline Setembro/2008 & 0,174 & 0,062 & 19,5 & Esférico & 1.194 & 34 & 0,74 \\
\hline Outubro/2008 & 0,175 & 0,070 & 20,0 & Esférico & 1.256 & 36 & 0,71 \\
\hline Novembro/2008 & 0,150 & 0,092 & 15,5 & Esférico & 754 & 21 & 0,62 \\
\hline Dezembro/2008 & 0,119 & 0,059 & 20,0 & Esférico & 1.256 & 36 & 0,67 \\
\hline Janeiro/2009 & 0,150 & 0,090 & 17,6 & Esférico & 973 & 28 & 0,62 \\
\hline Fevereiro/2009 & - & - & - & Aleatório & - & - & - \\
\hline Março/2009 & 0,056 & 0,050 & 21,5 & Esférico & 1.654 & 47 & 0,53 \\
\hline Abril/2009 & 0,172 & 0,069 & 28,0 & Esférico & 2.462 & 70 & 0,71 \\
\hline Maio/2009 & 0,170 & 0,075 & 23,5 & Esférico & 1.734 & 49 & 0,69 \\
\hline Junho/2009 & 0,080 & 0,107 & 20,7 & Esférico & 1.345 & 38 & 0,43 \\
\hline Julho/2009 & 0,083 & 0,027 & 15,5 & Esférico & 754 & 21 & 0,75 \\
\hline Agosto/2009 & 0,141 & 0,007 & 34,0 & Esférico & 3.630 & 104 & 0,95 \\
\hline
\end{tabular}

Observa-se que em onze avaliações (Tabela 1), apresentaram como melhor modelo ajustado o esférico. Este padrão é um forte indicativo de que a infestação da mosca negra se dá através de plantas vizinhas (fonte de inóculo) e vai crescendo até ocupar todo o pomar tendendo para aleatoriedade em altas infestações. O ajuste ao modelo esférico é o mais comum em estudos de insetos (Boareto \& Brandão, 2000; Farias et al., 2004) e se caracteriza por formar no campo "focos" ou "reboleiras" onde os mesmos se agrupam.
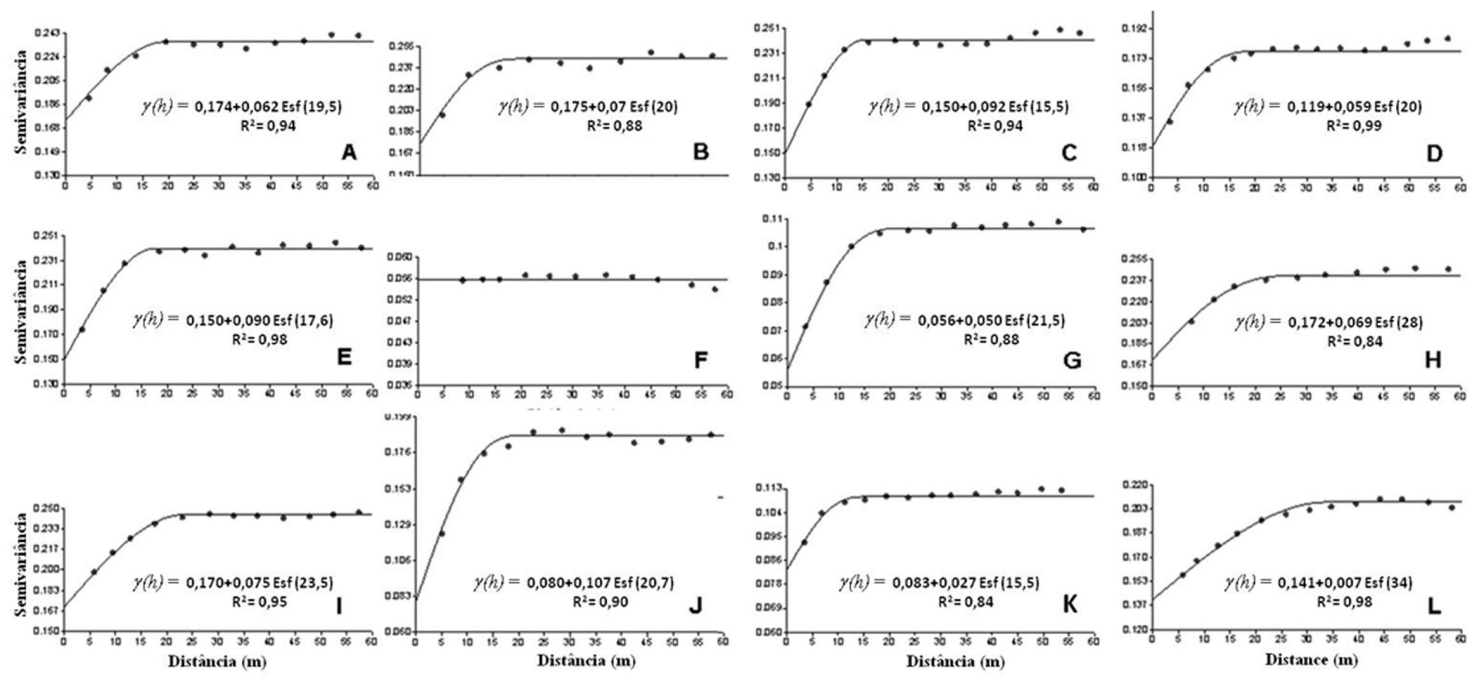

Figura 1. Semivariogramas da distribuição espacial da mosca-negra-dos-citros (A. woglumi), na área experimental. Fazenda Santana. Capitão Poço - PA. A: Setembro/2008; B: Outubro/2008; C: Novembro/2008; D: Dezembro/2008; E: Janeiro/2009; F: Fevereiro/2009; G: Março/2009; H: Abril/2009; I: Maio/2009; J: Junho/2009; K: Julho/2009; L: Agosto/2009 
A relação $k$ [Fração $C_{0} /\left(C_{0}+C_{1}\right)$ ] fornece uma medida para estimar quanto de aleatoriedade existem nos levantamentos, observou-se nas datas de amostragem, com exceção do mês de agosto, que os valores variaram de 0,43 a 0,75 I (Tabela 1), indicando que houve uma variação máxima de $75 \%$ nos levantamentos da mosca negra. Esses resultados estão dentro do recomendado por Jounel \& Huijbregts (1978), que citam que valores maiores que 0,80 indicam que o fenômeno estudado está tendendo para a aleatoriedade e que não existe nenhuma dependência entre as amostras.

Para o mês de fevereiro a população de A. woglumi encontrava-se bastante reduzida na área experimental, impossibilitando a detecção de dependência espacial, fenômeno este denominado de efeito pepita puro. Para este mês o modelo que melhor representou a distribuição da praga foi o aleatório. Dinardo-Miranda et al. (2007) com o objetivo de analisar a distribuição espacial de cigarrinha-das-raízes Mahanarva fimbriolata (Stal), também observaram efeito pepita puro nas primeiras avaliações devido a baixa população da praga, não permitindo assim verificar a dependência espacial. No entanto a partir da segunda geração com - aumento populacional da cigarrinha na área, verificou-se que esta se distribui de forma agregada na cultura da cana-de-açúcar e que esse padrão não se alterou ao longo do tempo, nem foi influenciado pelo nível de infestação.

A Figura 1 apresenta os semivariogramas das distribuições espaciais da mosca-negrados-citros na área experimental. O coeficiente de determinação $\left(R^{2}\right)$ indica a qualidade do ajuste do modelo do semivariograma teórico ao experimental. Valores próximos de 1 indicam bom ajuste, o que pode ser observado para - presente trabalho onde esse parâmetro apresentou amplitude de 0,84 a 0,99. Através dos parâmetros desses modelos, foram interpolados os levantamentos através da krigagem que nos forneceu mapas da distribuição espacial mostrando ás áreas de maior e menor infestação de mosca-negra-dos-citros na área do presente estudo (Figura 2).

Para as três primeiras avaliações (setembro, outubro e novembro), observou-se

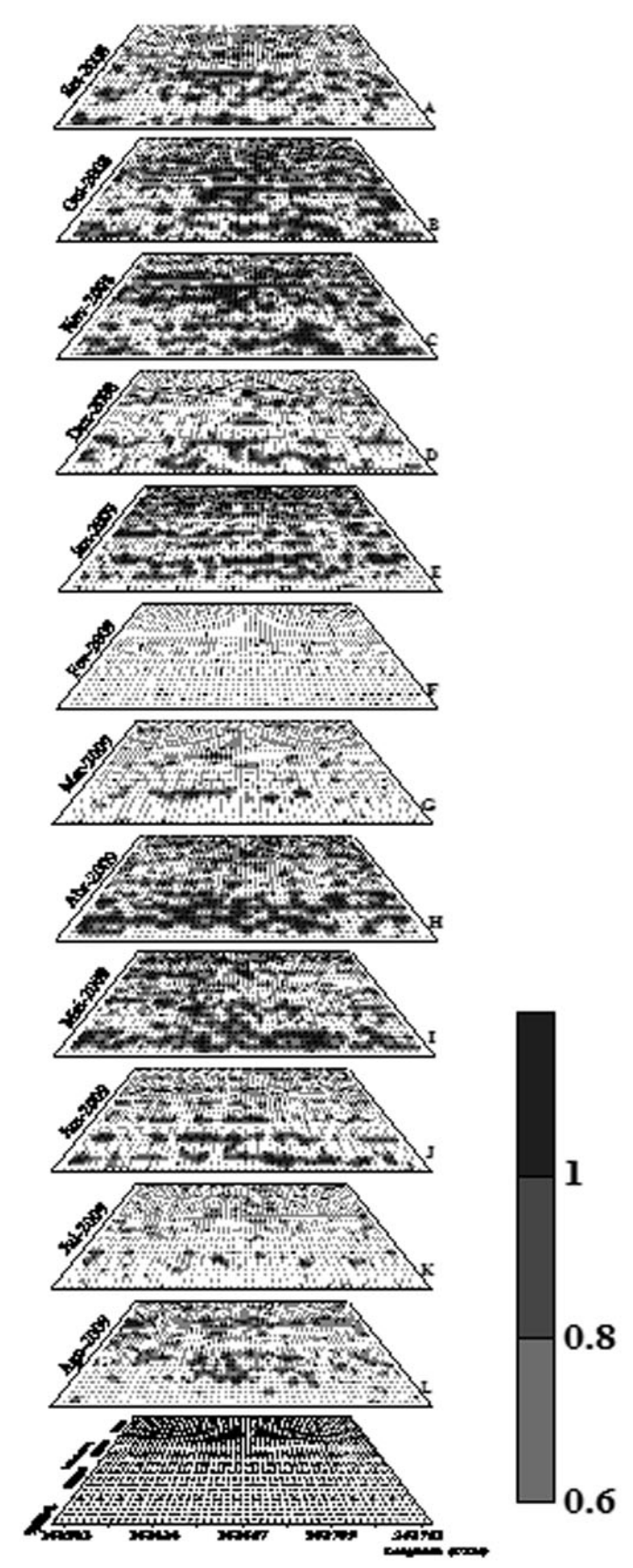

Figura 2. Mapas de krigagem para infestação de moscanegra-dos-citros (A. woglumi). Fazenda Santana, Capitão Poço - PA. A: Set/08; B: Out/08; C: Nov/08; D: Dez/08; E: Jan/09, F: Fev/09; G: Mar/09; H: Abr/09; I: Mai/09; J: Jun/09; L: Jul/09; M: Ago/09.

um crescimento gradativo da praga em estudo, como o mostrado nos mapas de krigagem (Figura 2); este aumento na infestação de mosca negra se deve, provavelmente, ao baixo índice pluviométrico nessa época do ano, aliada ao fato de não se efetuar qualquer controle químico na área. Este mesmo padrão de crescimento da infestação aliado a baixa precipitação pluviométrica foi observado por Medeiros et al. (2009) e Silva et al. (2011a) estudando a 
infestação de mosca-negra-dos-citros também sem a utilização de controle químico.

O mês de dezembro, ao contrario dos meses anteriores, apresentou redução do índice de plantas com presença de mosca-negra-doscitros, apresentando infestação de $24,8 \%$ de plantas na área experimental (Figura 3). O mapa de krigagem para este mês mostra claramente as reboleiras formadas, justificando o modelo (Figura 2). O mês de Janeiro já apresenta aumento da infestação, com crescimento considerável apresentando índice de $50,2 \%$ das plantas infestadas por mosca-negra-dos-citros
(Figura 3). Redução drástica foi observada no mês de fevereiro, onde apenas $103(6,2 \%)$ plantas de um total de 1.664 apresentavam-se infestadas (Figura 3). Esta redução pode está associada á elevada precipitação pluviométrica dos meses de janeiro e fevereiro. Flanders (1969) observou que ventos fortes e chuvas pesadas mostraramse capazes de desalojar e matar adultos da praga, enquanto Maia (2008), Medeiros et al. (2009) e Silva et al. (2011a) relatam que a população de A. woglumi é bastante reduzida devido a maior precipitação pluviométrica.

Devido à falta de dependência

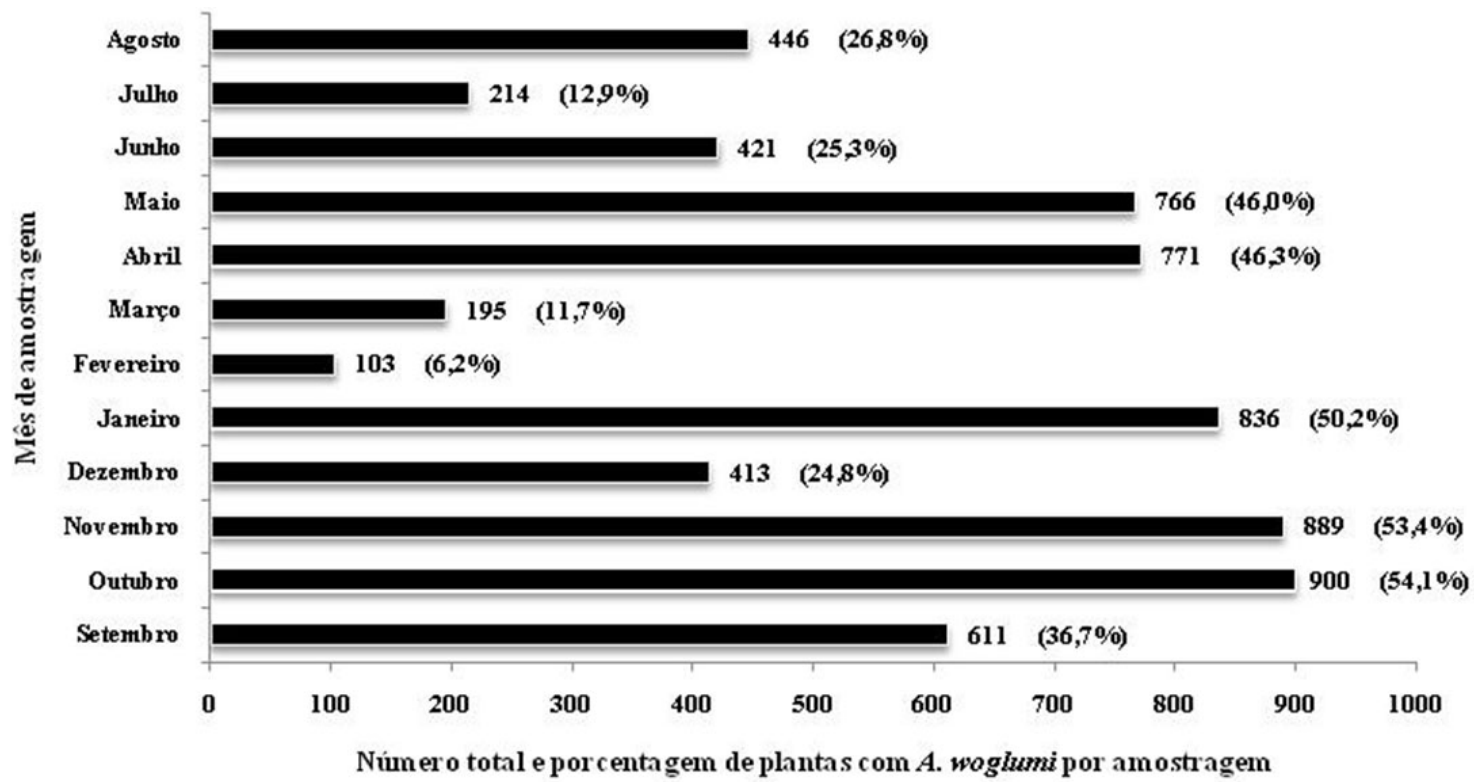

Figura 3. Número total e porcentagem de plantas com mosca-negra-dos-citros (A. woglumi) na área experimental, em cada mês de amostragem. Fazenda Santana, Capitão Poço - PA.

espacial para o mês de fevereiro, caracterizada nas análises variográficas, não foi realizada a krigagem para a infestação de mosca negra, pois não se detectou uma continuidade espacial que justificasse a aplicação da geoestatística para a referida estimação. Já para o mês de março, apesar de ser o mês de maior precipitação (447 mm), já apresenta um índice maior de plantas com mosca negra em relação ao mês anterior (fevereiro), infestação essa de $11,7 \%$ no presente estudo (Figura 3). Esse aumento possibilitou detectar a dependência espacial para o presente sistema de plantio cujo melhor modelo foi o esférico (Tabela 1) e a presença de pequenas agregações como observado no mapa de kigagem (Figura 2).

Os meses de fevereiro e março apresentaram menor infestação de mosca negra em relação ás doze avaliações feitas (Figura 3). Nas avaliações desse período foi possível observar reduzido número de ninfas, sendo a presença dos adultos o principal fator responsável pelas observações de presença de mosca-negra-dos-citros. Estes presentes em manchas ou reboleiras que atuam como focos de infestação que aliadas a indivíduos migrantes são responsáveis por novas infestações da área quando as condições novamente se mostrarem favoráveis.

Para abril e maio observou-se elevada infestação de mosca negra, logo após um período intenso de chuvas (janeiro, fevereiro e março) (Silva et al., 201 la), esse aumento está relacionado a um maior crescimento vegetativo 
das plantas de citros que se da nesse período de maior precipitação na Região Norte do país. Dessa forma o modelo esférico para os referidos meses justifica-se, dentre outros fatores, a esse período de intenso crescimento vegetativo, em que a praga concentra suas atividades em manchas (reboleiras) de árvores cítricas que oferecem condições adequadas para seu desenvolvimento. Para o mês de julho, observase pequena redução na infestação de mosca negra na área experimental (12,9\%), seguido por aumento da mesma para o mês de agosto (26,8\% de infestação) (Figura 3), como se observa nos mapas de krigagem (Figura 2), podendose inferir que a população de A. woglumi encontrou novamente condições favoráveis de desenvolvimento.

\section{Conclusões}

A distribuição espacial da moscanegra-dos-citros dá-se, predominantemente, em agrupamentos com dependência espacial descrita pelo modelo esférico, formando reboleiras de 15,5 a $34 \mathrm{~m}$ (alcance do modelo).

\section{Agradecimentos}

Ao Conselho Nacional de Ciência e Tecnologia - CNPa pela bolsa de mestrado concedida ao primeiro autor. A Fazenda Santana, Capitão Poço - PA, por disponibilizar a área para o desenvolvimento do experimento do presente trabalho.

\section{Referências}

Argov, Y., Rossler, Y., Voet, D. 1999. The Biology and phonology of the citrus whitefly Dialeuredes citri, on citrus in the coastal plain of Israel. Entomologia Experimemtalis et Applicata 93: 21-27.

Boaretto, M.A.C., Brandão, A.L.S. 2000. Amostragem de insetos. Universidade Estadual do Sudoeste Bahia, Departamento de Fitotecnia e Zootecnia área de Entomologia, Vitória da Conquista, BA. http://www. Uesb.br/ entomologia/amostrag.html. <Acesso em 07 de jan. 2010>.

Correia, R.G. Lima, A.C.S. Farias, P.R.S., Maciel, F.C.S., Silva, M.W., Silva, A.G. 2011. Primeiro registro da ocorrência de Mosca-Negra-dosCitros, Aleurocanthus woglumi Ashby, 1915 (Hemiptera: Sternorrhyncha: Aleyrodidae) em Roraima. Agro@mbiente 5: 245-248.

Dinardo-Miranda, L.L., Vasconcelos, A.C.M.,
Vieira, S.R., Fracasso, J.V., Grego, C.R. 2007. Uso da geoestatística na avaliação da distribuição espacial de Mahanarva fimbriolata em canade-açúcar. Bragantia 66: 449-455.

Dowell, R., Fitzpatrick, G.E. 1978. Effect of temperature on the growth and survival of the citrus blackfly. Canadian Entomologist 110: 13471350.

Farias, P.R.S., Roberto, S.R., Lopes, J.R.S., Perecin, D. 2004. Geostatistical characterization of the spatial distribution of Xylella fastidiosa sharpshooter vectors on citrus. Neotropical Entomology 33: 13-20.

Farias, P.R.S., Sánchez-Vila, X., Barbosa, J.C., Vieira, S.R., Ferraz, L.C.C.B., Solis-Delfin, J. 2002. Using geostatistical analysis to evaluluate the presence of Rotylenchulus reniformis in cotton crops in Brazil: economic implications. Journal of Nematology 34: 232-238.

Flanders, S.E. 1969. Observations on citrus blackfly parasites in índia and México and correlated circunstances. Canadian Entomologist 101: 467480.

IBGE. Instituto Brasileiro de Geográfia e Estatistica. Estados, lavoura permanente. 2012.http://www. ibge.gov.br/estadosat/temas.php? sigla=pa\&te ma=lavourapermanente2010. <Acesso em 17 de mar.2012>.

Journel, A.G., Huijbrebts, C.J. 1978. Mining geostatistics. Academic Press, Londres, UK. 600 p.

Leal, R.M., Barbosa, J.C, Costa, M.G., Belasque Junior, J., Yamamoto, P.T.; Dragone, J. 2010. Distribuição espacial de huanglongbing (greening) em citros utilizando a geoestatística. Revista Brasileira de Fruticultura 32: 808-818.

Levin D.A. 1973. The role of trichomes in plant defense. Quarterly Review of Biology 48: 15-21.

Liebhold, A.M., Rossi, R.E. Kemp, W.P. 1993. Geostatistic and geographic information system in applied insect ecology. Annual Review of Entomology 38: 303-327.

Maia, P.S.P. 2008. Caracterização da distribuição espacial da mosca negra dos citros (Aleurocanthus woglumi Ashby, 1915) em pomar georreferenciado para determinar um plano de amostragem sequencial. 77 f. Dissertação (Mestrado); Universidade Federal Rural da Amazônia, Belém.

MAPA. 2010. Ministério da Agricultura, Pecuária e Abastecimento. Lista de pragas quarentenárias presentes - (A2). Disponivel em:< http://extranet. agricultura.gov.br/sislegisconsulta/servlet/ VisualizarAnexo?id=14644. <Acesso em 23 de out. 2010>. 
Medeiros, F.R., Lemos, R.N.S., Ottati, A.L.T., Araújo, J.R.G., Machado, K.K.G., Rodrigues, A.A.C. 2009. Dinâmica populacional da mosca-negra-doscitros Aleurocanthus woglumi Ashby (Hemiptera: Aleyrodidae) em Citrus spp. no município de São Luís - MA. Revista Brasileira de Fruticultura 31: 1016-1021.

Monteiro, B.S., Rodrigues, K.C.V., Silva, A.G., Barros, R. 2012. Ocorrência da Mosca-negra-doscitros, Aleurocanthus woglumi Ashby (Hemiptera: Aleyrodidae) em Pernambuco. Revista Caatinga 25: $173-176$.

Moreira, R.Y.O., Arruda, M.S.P., Arruda, A.C., Santos, L.S., Müller, A.H.; Giulhon, M.S.P., Santos, A.S., Terezo, E. 2006. Antraquinonas e naftoquinonas do caule de um espécime de reflorestamento de Tectona grandi (Verbenaceae). Revista Brasileira de Farmacognosia 16: 392-396.

Parkinson, K., Seales, J. 2000. Citrus blackfly, its presence and management in Trinidad and Tobago. Procaribe News, Network, 11 p.

Pasek J.E. 1988. Influence of wind and windbreaks on local dispersal of insects. Agriculture, Ecosystems and Environment 22: 539-554.

Pena, M.R., Vendramim, J.D., Lourenção, A.L., Silva, N.M., Yamamoto, P.T., Gonçalves, M.S. 2008. Ocorrência da mosca-negra-dos-citros, Aleurocanthus woglumi Ashby (Hemiptera: Aleyrodidae) no estado de São Paulo. Revista de Agricultura 83: 61-65.

Root, R.B. 1973. Organization of a plant-arthropod association in simple and diverse habitats: the fauna of collards (Brassica oleracea). Ecological Monographs 43: 95-124.

Silva, A.B. 2005. Mosca negra dos citros, Aleurocanthus woglumi Ashby, praga potencial para a citricultura brasileira. In: Poltronieri, L.S., Trindade, D.R., Santos, I.P. Pragas e doenças de cultivos amazônicos. p. 147-156. Embrapa, Belém, 484p.

Silva, A.G, Boiça Junior, A.L., Farias, P.R.S., Barbosa, J.C. 2011 a. Infestação da mosca-negra-doscitros em pomares de citros em sistema de plantio convencional e agroflorestal. Revista Brasileira de Fruticultura 33: 53-60.

Silva, A.G., Farias, P.R.S., Boiça Junior, A.L., Correia, R.G., Silva, J.B., Rodrigues, N.E.L. 2011 b. Análise espacial da mosca-negra-dos-citros em pomar de citros utilizando a geoestatística. Revista de Agricultura 86: 102-114.

Soto, A., Ohlenschläeger, F., García-Marí, F. 2002. Distribution and sampling of the whiteflies Aleurothrixus floccosus, Dialeurodes citri, and Parabemisia myricae (Homoptera: Aleyrodidae) in Citrus in Spain. Journal of Economic Entomology
95: 167-173.

Vieira, S.R., Hatfield, T.L., Nielsen, D.R., Biggar, J.W. 1983. Geostatistical theory and application to variability of some agronomical properties. Hilgardia 51: 1-75.

Yared, J.A.G., Brienza Júnior, S., Marques, L.C.T. 1998. Agrossilvicultura: conceitos, classificação e oportunidades para a aplicação na Amazônia brasileira. Belém: Embrapa-CPATU, (EmbrapaCPATU. Documento, 104), 39 p. 